\title{
A DATA-DRIVEN, TWO-TEMPERATURE SOLAR WIND MODEL WITH ALFVÉN WAVES
}

\author{
B. van der Holst ${ }^{1}$, W. B. Manchester IV ${ }^{1}$, R. A. Frazin ${ }^{1}$, A. M. VÁsquez ${ }^{2}$, G. Tóth ${ }^{1}$, And T. I. Gombosi ${ }^{1}$ \\ ${ }^{1}$ Atmospheric Oceanic and Space Sciences, University of Michigan, Ann Arbor, MI 48109, USA \\ ${ }^{2}$ Instituto de Astronomía y Física del Espacio (CONICET-UBA) and FCEN (UBA), CC 67, Suc 28, Ciudad de Buenos Aires, Argentina \\ Received 2010 July 13; accepted 2010 October 15; published 2010 November 24
}

\begin{abstract}
We have developed a new three-dimensional magnetohydrodynamic (MHD) solar wind model coupled to the Space Weather Modeling Framework (SWMF) that solves for the different electron and proton temperatures. The collisions between the electrons and protons are taken into account as well as the anisotropic thermal heat conduction of the electrons. The solar wind is assumed to be accelerated by the Alfvén waves. In this paper, we do not consider the heating of closed magnetic loops and helmet streamers but do address the heating of the protons by the Kolmogorov dissipation of the Alfvén waves in open field-line regions. The inner boundary conditions for this solar wind model are obtained from observations and an empirical model. The Wang-Sheeley-Arge model is used to determine the Alfvén wave energy density at the inner boundary. The electron density and temperature at the inner boundary are obtained from the differential emission measure tomography applied to the extreme-ultraviolet images of the STEREO $A$ and $B$ spacecraft. This new solar wind model is validated for solar minimum Carrington rotation 2077 (2008 November 20 through December 17). Due to the very low activity during this rotation, this time period is suitable for comparing the simulated corotating interaction regions (CIRs) with in situ $A C E$ /WIND data. Although we do not capture all MHD variables perfectly, we do find that the time of occurrence and the density of CIRs are better predicted than by our previous semi-empirical wind model in the SWMF that was based on a spatially reduced adiabatic index to account for the plasma heating.
\end{abstract}

Key words: interplanetary medium - magnetohydrodynamics (MHD) - methods: numerical - solar wind - Sun: corona

Online-only material: color figures

\section{INTRODUCTION}

Understanding the global three-dimensional (3D) structure of the solar wind and interplanetary space is vital for space weather predictions. For the numerical forecasting of the time of arrival and impact at Earth of eruptive events from the Sun, the development of realistic models of the solar corona (SC) and inner heliosphere (IH) is needed. Several 3D models have recently been developed (e.g., Mikić et al. 1999; Roussev et al. 2003; Usmanov \& Goldstein 2006; Cohen et al. 2007; Feng et al. 2007; Lionello et al. 2009) for the simulation of the global solar wind structure.

The physical processes responsible for the acceleration and heating of the solar wind plasma are still not known. Alfvén waves have been suggested as one of the possible mechanisms (Belcher 1971; Jacques 1977). In the Alfvén wave/turbulence type of solar wind models, both key questions, how to accelerate and how to heat, are closely connected. The wave pressure can accelerate the solar wind while the gradual dissipation of the waves can heat the plasma (Hollweg 1986). Suzuki (2006) constructed a one-dimensional Alfvén wave solar wind model that predicts the solar wind speed in the vicinity of Earth. Several two-dimensional (2D) coronal models containing Alfvén waves have been created over the years (see, e.g., Lou 1994; Ofman \& Davila 1995; Bravo \& Stewart 1997; Ruderman et al. 1998). Two-dimensional global axisymmetric solar wind models with Alfvén waves were first developed by Usmanov et al. (2000). More recent Alfvén wave driven models include the partial reflection back down to the Sun (see, e.g., Chandran \& Hollweg 2009a; Cranmer 2010; Verdini et al. 2010) while Evans et al. (2009) investigated surface Alfvén waves in the 3D solar wind. Sokolov et al. (2009) present the frequency-resolved transport equations for the Alfvén waves and how they couple to the magnetohydrodynamic (MHD) equations for an arbitrary 3D domain.

Hartle \& Sturrock (1968) were the first who realized that the collisions between the electrons and protons at 2 solar radii (and farther) from the Sun are infrequent, so that the two species can have quite different temperatures. More recently, this two-species approach for electrons and protons was extended by Tu \& Marsch (1997), Laitinen et al. (2003), and Vainio et al. (2003) with Alfvén waves for the heating and acceleration of the solar wind plasma. In these papers, onedimensional models with a frequency-resolved Alfvén wave spectrum were derived. Two-dimensional, two-species simulations without Alfvén waves were performed by Endeve et al. (2004). The 2D two-temperature simulations of Hu et al. (2003a, 2003b) are with Alfvén waves, which demonstrate quite good agreement with in situ Ulysses observations of the proton velocity, density, temperature, and flux.

Recently, several 3D solar wind models have been developed that start from the chromosphere and extend well into the corona, see, e.g., Lionello et al. (2009) and Downs et al. (2010). These models start from either uniform prescribed plasma density and temperature at the chromosphere or obtain the inner boundary density from the so-called radiative energy balance model (Withbroe 1988) at the $0.5 \mathrm{MK}$ temperature level. These models are able to produce simulated images of the extreme ultraviolet (EUV) and the soft X-ray, which can then be compared with observations. In this paper, we adopt a different strategy, namely, starting the model at $r=1.035 R_{\odot}$ (solar radius) where the temperature is in the $1 \mathrm{MK}$ range and thereby avoiding the computationally expensive chromosphere and transition region. However, this means that we need to obtain the density and 
temperature by other means. In this paper, we use the differential emission measure tomography (DEMT) applied to EUV images as developed by Frazin et al. (2009) and Vásquez et al. (2010) to determine the density and electron temperature at the inner boundary. In this solar wind model, the onset of coronal mass ejections (CMEs) could still be simulated by, for instance, coupling the flux emergence model of the convection zone (Fang et al. 2010) to the solar wind.

This paper is organized as follows. In Section 2, we describe the two-temperature MHD model with Alfvén waves and the method to obtain the boundary conditions from observations. In Section 3, we validate our solar wind model for Carrington rotation 2077. We conclude with a summary in Section 4.

\section{SOLAR WIND MODEL}

In this section, we describe the details of the solar wind model. The two-temperature MHD equations for the electrons and protons are used. They include anisotropic thermal heat conduction for the electrons and Alfvén waves to heat and accelerate the protons. The method to obtain the inner coronal boundary conditions for a solar wind using a synoptic magnetogram and DEMT is explained in Section 2.2

\subsection{Governing Equations}

The gross macroscopic properties of the solar wind can be well described by the MHD equations. In the inertial frame, the time-dependent behavior of the fully ionized hydrogen plasma that we assume in our model is described by (see, e.g., Braginskii 1965 for a derivation of the two-fluid equations):

$$
\begin{gathered}
\frac{\partial \rho}{\partial t}+\nabla \cdot(\rho \mathbf{u})=0 \\
\rho \frac{\partial \mathbf{u}}{\partial t}+\rho \mathbf{u} \cdot \nabla \mathbf{u}=-\rho \frac{G M_{\odot}}{r^{3}} \mathbf{r}-\nabla\left(p_{\mathrm{p}}+p_{\mathrm{e}}\right) \\
+\frac{1}{\mu_{0}}(\nabla \times \mathbf{B}) \times \mathbf{B}-\nabla p_{\mathrm{w}},
\end{gathered}
$$

$\frac{\partial p_{\mathrm{p}}}{\partial t}+\nabla \cdot\left(p_{\mathrm{p}} \mathbf{u}\right)=(\gamma-1)\left[-p_{\mathrm{p}} \nabla \cdot \mathbf{u}+\frac{2}{\tau_{\mathrm{pe}}}\left(p_{\mathrm{e}}-p_{\mathrm{p}}\right)+Q_{\mathrm{p}}\right]$,

$$
\frac{\partial p_{\mathrm{e}}}{\partial t}+\nabla \cdot\left(p_{\mathrm{e}} \mathbf{u}\right)=(\gamma-1)\left[-p_{\mathrm{e}} \nabla \cdot \mathbf{u}+\frac{2}{\tau_{\mathrm{pe}}}\left(p_{\mathrm{p}}-p_{\mathrm{e}}\right)-\nabla \cdot \mathbf{q}_{\mathrm{e}}\right],
$$

$$
\frac{\partial \mathbf{B}}{\partial t}-\nabla \times(\mathbf{u} \times \mathbf{B})=0,
$$

where $\rho$ is the mass density, $\mathbf{u}$ is the velocity (of both the protons and electrons), $p_{\mathrm{e}}$ and $p_{\mathrm{p}}$ are the electron and proton pressures, respectively, $T_{\mathrm{e}}$ and $T_{\mathrm{p}}$ are the electron and proton temperatures, respectively, $\mathbf{B}$ is the magnetic field, $m_{\mathrm{p}}$ is the proton mass, $G$ is the gravitational constant, $M_{\odot}$ is the solar mass, and $\mu_{0}$ is the permeability of vacuum. The adiabatic index, $\gamma$, is fixed at $5 / 3$. We assume that the solar wind plasma can be described as a fully ionized hydrogen plasma that is quasi-neutral, so that the electron and proton number densities are equal: $n_{\mathrm{e}}=n_{\mathrm{p}}$. We also assume that the electron and proton velocities are equal by omitting the Hall term in the two-fluid equations. The second term on the right-hand side of the proton and electron pressure equations (3) and (4) represents the collisional heat transfer between the protons and electrons, established on the timescale

$$
\tau_{\mathrm{pe}}=\frac{2}{3} \frac{m_{\mathrm{p}}}{\eta e^{2} n_{\mathrm{e}}}
$$

where $e \approx 1.6 \times 10^{-19}$ is the elementary charge and $\eta$ is the resistivity. The latter depends on the Coulomb logarithm which we assume $\ln \Lambda=20$ uniformly. The electrons and protons are only collisionally coupled near the Sun. For the electron thermal heat flux, we use the collisional formulation of Spitzer:

$$
\mathbf{q}_{\mathrm{e}}=-\kappa_{\mathrm{e}} T_{\mathrm{e}}^{5 / 2} \frac{\mathbf{B B}}{B^{2}} \cdot \nabla T_{\mathrm{e}},
$$

where $\kappa_{\mathrm{e}} \approx 9.2 \times 10^{-12} \mathrm{~W} \mathrm{~m}^{-1} \mathrm{~K}^{-7 / 2}$ again assuming $\ln \Lambda=20$. The collisional formulation of the heat flux is used between $1 R_{\odot}$ (solar radius) and $5 R_{\odot}$. Between $5 R_{\odot}$ and $10 R_{\odot}$ the heat flux is smoothly diminished to zero. We do not consider using the collisionless heat conduction formulation of Hollweg (1978) in the two-temperature solar wind model in this paper. Beyond $10 R_{\odot}$, the electron heat flux is set to zero. The heating of protons $Q_{\mathrm{p}}$ and the acceleration by the gradient in the wave pressure $p_{\mathrm{w}}$ is described by the evolution of the Alfvén waves. In this paper, we will use the Wentzel-Kramers-Brillouin (WKB) approximation for the short wavelength Alfvén waves (Jacques 1977). The time evolution of the Alfvén wave energy density is given by

$$
\frac{\partial E_{\mathrm{w}}}{\partial t}+\nabla \cdot\left[E_{\mathrm{w}}\left(\mathbf{u}+\mathbf{u}_{\mathrm{A}}\right)\right]=-p_{\mathrm{w}} \nabla \cdot \mathbf{u}-Q,
$$

where $p_{\mathrm{w}}=E_{\mathrm{w}} / 2$ is the Alfvén wave pressure and $\mathbf{u}_{\mathrm{A}}=$ $\pm \mathbf{B} / \sqrt{\mu_{0} \rho}$ is the Alfvén speed. There are two Alfvén wave solutions, indicated by the \pm sign, corresponding to waves propagating in opposite direction. For the wave dissipation $Q$, we will use the phenomenological description of the Kolmogorov dissipation of the Alfvén waves (Hollweg 1986) to heat the protons in the pressure equation (3):

$$
Q_{\mathrm{p}}=Q=\frac{\left(E_{\mathrm{w}}\right)^{3 / 2}}{L \sqrt{\rho}}
$$

where $L=C / \sqrt{B}$ is the correlation length of the Alfvén waves and $C$ is a parameter that can be adjusted freely for a best model fit with observational data, e.g., the density at 1 AU.

In our solar wind model, we only consider Alfvén waves that are propagating away from the Sun with no reflection. Kolmogorov-like dissipation, however, only occurs when there are also counterpropagating Alfvén waves (see, e.g., Dmitruk et al. 2002; Cranmer \& van Ballegooijen 2005; Chandran et al. $2009 b$ ). These counterpropagating waves are usually assumed to arise by partial reflection due to inhomogeneities within the corona. This means that strictly speaking the Kolmogorov-type dissipation, given by Hollweg (1986), severely overestimates the amount of heating in the corona. On top of that, it is currently still an open issue how the turbulent energy cascade is partitioned between the protons and electrons (see, e.g., Stawarz et al. 2009; Breech et al. 2009). Due to the significant uncertainty in the fraction of the turbulent heating that is given to the electrons, we set the electron heating to zero for now and leave the general case for future work.

The set of two-fluid equations (1)-(8) is written in the primitive form. For the actual numerical implementation, the 
momentum and proton pressure equations, Equations (2) and (3), respectively, are transformed to the (near) conservative form for the momentum density and total energy density

$$
\begin{aligned}
& \frac{\partial \rho \mathbf{u}}{\partial t}+\nabla \cdot\left[\rho \mathbf{u u}+\left(p_{\mathrm{p}}+p_{\mathrm{e}}+p_{\mathrm{w}}+\frac{B^{2}}{2 \mu_{0}}\right) I-\frac{1}{\mu_{0}} \mathbf{B B}\right] \\
& \quad=-\rho\left[\frac{G M_{\odot}}{r^{3}} \mathbf{r}+\boldsymbol{\Omega} \times(\boldsymbol{\Omega} \times \mathbf{r})+2 \boldsymbol{\Omega} \times \mathbf{u}\right], \\
& \frac{\partial \mathcal{E}}{\partial t}+\nabla \cdot\left[\left(\mathcal{E}+p_{\mathrm{p}}+p_{\mathrm{e}}+p_{\mathrm{w}}+\frac{B^{2}}{2 \mu_{0}}\right) \mathbf{u}-\frac{1}{\mu_{0}} \mathbf{B B} \cdot \mathbf{u}+\mathbf{q}_{e}+E_{\mathrm{w}} \mathbf{u}_{A}\right] \\
& \quad=-\rho \mathbf{u} \cdot\left[\frac{G M_{\odot}}{r^{3}} \mathbf{r}+\boldsymbol{\Omega} \times(\boldsymbol{\Omega} \times \mathbf{r})\right],
\end{aligned}
$$

where

$$
\mathcal{E}=\frac{\rho u^{2}}{2}+\frac{p_{\mathrm{p}}+p_{\mathrm{e}}}{\gamma-1}+\frac{B^{2}}{2 \mu_{0}}+E_{\mathrm{w}},
$$

is the total energy density, and $\Omega$ is the angular velocity of the Sun. We use a uniform solar rotation with a sidereal period of 25.38 days, so that $\Omega=2.865 \times 10^{-6} \mathrm{rad} \mathrm{s}^{-1}$. These equations are solved in the frame corotating with the Sun, so that the Coriolis and centrifugal forces have been added to the momentum equation and the work of the centrifugal force to the energy equation. The time evolution equations (1), (10), (11), (5), (4), and (8) are solved with the numerical shock-capturing solvers of the MHD BATS-R-US code (Powell et al. 1999). In the numerical implementation, we solve for two Alfvén wave energy densities instead of a single $E_{w}: E_{w}^{+}$for the propagating Alfvén waves in the direction of $\mathbf{B}$ and $E_{w}^{-}$for the waves propagating antiparallel to $\mathbf{B}$.

\subsection{Data-driven Boundary Conditions}

The inner boundary conditions are specified as follows. We fix the mass density and electron temperature with the density and electron temperature obtained from the DEMT as described in Section 2.2.2. The radial component of the magnetic field, $B_{\mathrm{r}}$, is fixed with the potential field source surface (PFSS) magnetic field (Section 2.2.1). The tangential components of the magnetic field are extrapolated with zero radial gradient. In the frame corotating with the Sun, we use a zero gradient extrapolation for the velocity component along the field lines. The boundary condition for the proton temperature is subtle. In general, fixing the mass density and electron temperature will give a pressure imbalance between the foot points of magnetic loops, which could result in syphon flows along the closed magnetic field lines, see for instance Cargill \& Priest (1980) and Harra et al. (2004). If we fix the proton temperature to the electron temperature values, then the proton and electron pressure equations (3) and (4) cannot settle to a steady state. To ensure we can obtain a steady state solar wind, the proton temperature is extrapolated to accommodate the pressure buildup at the footpoints of the coronal loops. In this paper, we will not further address syphon flows, since our main goal is the development of the solar wind model. The energy density of the Alfvén waves is set to zero at the inner boundary of the helmet streamers and other closed field-line regions. This prevents the closed field lines from opening up. In the open fieldline regions, we fix the outward propagating Alfvén wave energy via the Wang-Sheeley-Arge (WSA) method (Section 2.2.3). The wave energy density, $E_{\mathrm{w}}$, of the waves that propagate back to the Sun is extrapolated.



Figure 1. Carrington map of the radial magnetic field component at $r=$ $1.035 R_{\odot}$. This map is obtained via a finite difference PFSS solution using a GONG synoptic magnetogram for Carrington rotation 2077.

(A color version of this figure is available in the online journal.)

At the outer boundary the flow is faster than the fast magnetoacoustic speed so that all characteristic waves excited at the outer boundary will leave the domain. In other words, no information from outside of the domain will propagate into the domain. We can therefore extrapolate with zero gradient all the MHD variables at the outer boundary.

\subsubsection{Finite Difference Iterative Potential-field Solver}

For the radial magnetic field, the synoptic GONG (Global Oscillation Network Group) map for Carrington Rotation (CR) 2077 (2008 November 20 through December 17) is used. To obtain the radial magnetic field at the inner boundary at $r=1.035 R_{\odot}$ of the computational domain instead of the photospheric level, we assume a potential (curl-free) field that matches the magnetogram field at the photosphere, while a purely radial magnetic field is enforced at the source surface, $r=R_{\mathrm{SS}}=2.5 R_{\odot}$. In other words, we solve for the Laplace equation for the scalar potential $\Phi=\Phi(r, \vartheta, \varphi)$ :

$$
\nabla \cdot(\nabla \Phi)=0
$$

subject to the boundary conditions $\partial \Phi / \partial r\left(r=R_{\odot}\right)=B_{0 \mathrm{r}}$ and $\Phi\left(r=R_{\mathrm{SS}}\right)=0$. The solution of this equation is called the PFSS solution (Schatten et al. 1969; Altschuler \& Newkirk $1969)$. Once the potential is found, the magnetic field is given by $\mathbf{B}_{0}=\nabla \Phi$. This PFSS problem is discretized using a staggered spherical grid. The magnetic field is discretized on the cell faces and the potential is located on the cell centers. We use the Krylov-type iterative method Bi-CGSTAB (van der Vorst 1992) to find the solution. Using a finite difference method instead of a spherical harmonic expansion has the advantage that it is better behaved near regions of strong magnetic field (no ringing patterns). For more information about the finite difference iterative potential field solver and the comparison with spherical harmonic expansion method, see Tóth et al. (2010). A Carrington map of the radial magnetic field component at the inner coronal boundary $r=1.035 R_{\odot}$ is shown in Figure 1 for CR2077. This rotation is a solar minimum configuration with only two weak flux concentration.

The PFSS solution for $\mathbf{B}_{0}$ is used for the initial condition. In the coronal model, we assume that the PFSS field $\mathbf{B}_{0}$ between the source surface radius and the outer boundary is radial. More importantly, in the numerical solver of the solar wind model, we split the magnetic field as $\mathbf{B}=\mathbf{B}_{0}+\mathbf{B}_{1}$ as described by Tanaka (1994), so that we only need to numerically solve for the change in the magnetic field $\mathbf{B}_{1}$ compared to the intrinsic field $\mathbf{B}_{0}$ given by the PFSS and the radial field beyond the source surface. 
The advantage of this splitting is that the solver is inherently more accurate for a small $\mathbf{B}_{1}$ then for the full magnetic field, which reduces the numerical diffusion (Tanaka 1994). In this splitting scheme, no assumption is made that $\mathbf{B}_{1}$ should be small compared to $\mathbf{B}_{0}$. The inner boundary condition for $B_{\mathrm{r}}$ reduces to $B_{1 \mathrm{r}}=0$ at $r=1.035 R_{\odot}$. That we use the PFSS field for $\mathbf{B}_{0}$ does not mean that the obtained topology of the solar wind field $\mathbf{B}_{0}+\mathbf{B}_{1}$ will be similar to that of the PFSS. The solar wind will have for instance different-sized streamers, does not have a source surface, and does have a Parker spiral due to the solar rotation.

\subsubsection{Differential Emission Measure Tomography}

EUV images of the Sun have been used to derive the differential emission measure (DEM) distributions (Howard et al. 2008). This distribution gives a measure of the amount of plasma as a function of the temperature in a given volume of space. Since these images are 2D projections along the line of sight of the 3D corona, the DEM corresponds to an integral of the plasma along the line of sight. To undo the projection effects, a recently developed technique applies tomography on a time series of EUV images under the assumption of no time variation and uniform solar rotation (Frazin et al. 2009) to derive a 3D DEM distribution. The technique, named DEMT, provides the 3D emissivity distribution in each EUV band. Local differential emission measure (LDEM) analysis is subsequently performed on each DEMT volume element, also known as a voxel, independently. The first two moments of the LDEM analysis (Equations (4) and (5) in Vásquez et al. 2010) will then give the $3 \mathrm{D}$ distribution of the electron density and temperature. The tomographic method is a linear inversion that assumes an optically thin plasma. For more details on this method, the reader is referred to Frazin et al. (2009).

The boundary conditions for the mass density and electron temperature are obtained from the DEMT method applied to CR2077 EUV images taken by the Extreme UltraViolet Imager (EUVI; Howard et al. 2008) on the Solar Terrestrial Relations Observatory (STEREO) $A$ and $B$ spacecraft. The tomographic reconstruction then provides the $3 \mathrm{D}$ emissivity in each EUVI coronal band (171, 195, and $284 \AA$ ), as described in Vásquez et al. (2010), in the height range 1.035-1.225 $R_{\odot}$. The LDEM moments for CR2077 are obtained under the DEMT assumption that the corona is in steady state during the EUVI data gathering time. The use of both STEREO satellites in Vásquez et al. (2010) reduced the time required to collect the data, compared to the full Carrington rotation of data needed when using a single satellite. During this period, the two spacecrafts were separated by $85.4 \pm 1.2$, which allowed for the reconstruction to be performed with data gathered in about $3 / 4$ of a Carrington rotation. This reduction diminishes the effect of coronal dynamics in the reconstructions. In addition, some image data points are rejected due to the need for optically thin plasma for the tomographic method, implying that $1.035 R_{\odot}$ is the lowest usable height for the results. The CR2077 DEMT data set we use here uses an angular grid of 180 longitudes and 90 latitudes. At height $1.035 R_{\odot}, 1.3 \%$ of the data points were rejected due to the coronal dynamic artifacts. To obtain a fully covered map for the inner coronal boundary conditions of the solar wind model, we use Delaunay triangulation to fill in the rejected data points with interpolated values. Figure 2 shows the Carrington maps of the final interpolated electron density and temperature for $r=1.035 R_{\odot}$.

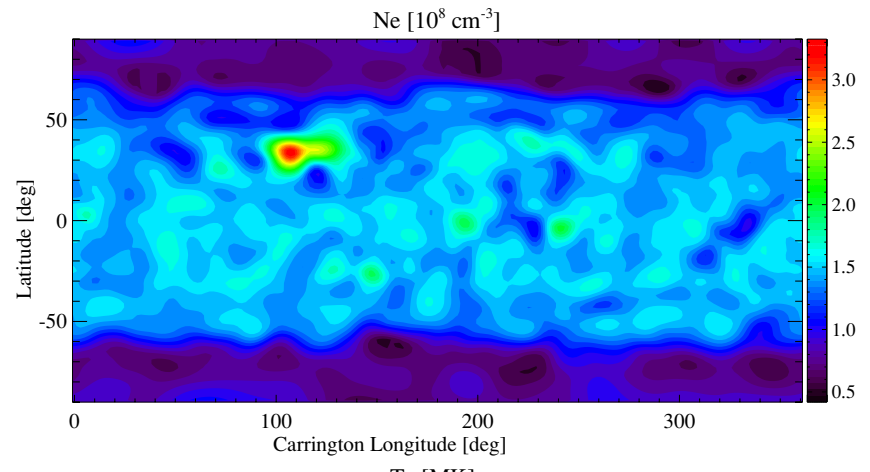

Te $[\mathrm{MK}]$

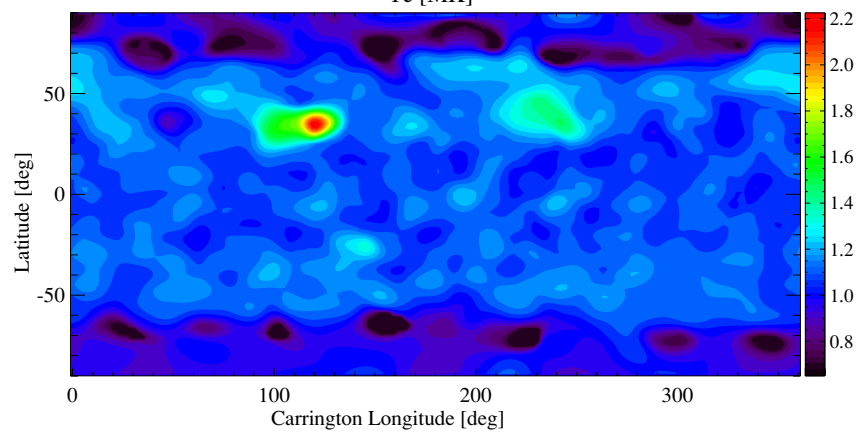

Figure 2. Carrington maps of the electron number density (top panel) and electron temperature (bottom panel) at $r=1.035 R_{\odot}$ obtained from the LDEM analysis.

(A color version of this figure is available in the online journal.)

\subsubsection{Wang-Sheeley-Arge Model}

To close the set of boundary conditions, we have to fix the Alfvén wave energy density at the inner coronal boundary. To the best of our knowledge, there are no data sources available that would directly give a 2D latitude-longitude map of the Alfvén wave energy at say $r=1.035 R_{\odot}$. However, this distribution of Alfvén wave energy is crucial for our solar wind model, since the waves that are launched from the inner boundary have to provide the wave pressure acceleration in the high speed wind. Below, we will outline a procedure that relates the Alfvén wave density at the inner boundary to the velocity at $1 \mathrm{AU}$ obtained from the empirical WSA model (Arge \& Pizzo 2000) using a one-dimensional approximation along the magnetic flux tubes of the PFSS field. We use the WSA speed at 1 AU only to determine the Alfvén wave energy at the inner boundary. Once this wave energy is known, the WSA speed is no longer needed in the model and instead the 1 AU velocity is subsequently selfconsistently calculated using the 3D Alfvén wave driven solar wind model. It should be pointed out that the field lines of the solar wind model in general do not have the same topology as the field lines of the PFSS model, and streamers can be different in size so that the acceleration along field lines of the wind model is not guaranteed to reproduce the velocity at $1 \mathrm{AU}$ as the WSA model.

The velocity distribution at $1 \mathrm{AU}$ can be obtained from the WSA model (Arge \& Pizzo 2000). Given the PFSS solution, the flux tube expansion factor $f_{\mathrm{s}}$ at any given colatitude $\vartheta$ and Carrington longitude $\varphi$ is defined as

$$
f_{\mathrm{S}}(\vartheta, \varphi)=\frac{\left|B_{r 0}\left(R_{\odot}, \vartheta, \varphi\right)\right| R_{\odot}^{2}}{\left|B_{r 0}\left(R_{\mathrm{SS}}, \vartheta_{\mathrm{SS}}, \varphi_{\mathrm{SS}}\right)\right| R_{\mathrm{SS}}^{2}},
$$

where $B_{r 0}$ is the radial magnetic field component of the flux tube. We ray trace the flux tube starting from $\left(R_{\odot}, \vartheta, \varphi\right)$ in the 
open field-line regions to the source surface. The coordinates of the intersection of this flux tube with the source surface are given by $\left(R_{\mathrm{SS}}, \vartheta_{\mathrm{SS}}, \varphi_{\mathrm{SS}}\right)$. In Arge \& Pizzo (2000), the velocity at $1 \mathrm{AU}$ from the WSA model was only dependent on $f_{\mathrm{s}}$. In Arge et al. (2003), the WSA model was further refined by letting the $1 \mathrm{AU}$ velocity also depend on the minimum angular distance $\theta_{\mathrm{b}}$ (in degrees) of the magnetic field foot point at the photosphere to the coronal hole boundary of the PFSS field. In our solar wind model, we use a more recent formulation of the WSA model suitable for GONG magnetograms (N. Arge 2010, private communication). Here, the empirical velocity at $1 \mathrm{AU}$ is given by

$$
u_{1 \mathrm{AU}}=240+\frac{675\left\{1-0.8 e^{\left[-\left(\frac{\theta_{\mathrm{b}}}{2.8}\right)^{1.25}\right]}\right\}^{3}}{\left(1+f_{\mathrm{s}}\right)^{1 / 4.5}}
$$

in units of $\mathrm{km} \mathrm{s}^{-1}$. In the WSA model formulation we use, the velocity is assigned at the source surface and assumed to propagate ballistically from this radius to $1 \mathrm{AU}$.

To obtain the Alfvén wave pressure at the inner coronal boundary from the WSA velocity at $1 \mathrm{AU}$, we trace the total energy from $1 \mathrm{AU}$ back to the Sun along the field lines of the PFSS model. The energy equation (11) can be rewritten as

$$
\begin{aligned}
& \frac{\partial}{\partial t}\left(\mathcal{E}-\rho \frac{G M_{\odot}}{r}\right)+\nabla \cdot\left[\left(\frac{\gamma}{\gamma-1}\left(p_{\mathrm{p}}+p_{\mathrm{e}}\right)+\frac{1}{2} \rho u^{2}\right.\right. \\
& \left.\left.-\rho \frac{G M_{\odot}}{r}+\frac{3}{2} E_{\mathrm{w}}\right) \mathbf{u}-\frac{1}{\mu_{0}}(\mathbf{u} \times \mathbf{B}) \times \mathbf{B}+\mathbf{q}_{\mathrm{e}}+E_{\mathrm{w}} \mathbf{u}_{\mathrm{A}}\right]=0,
\end{aligned}
$$

in the inertial frame and includes the gravitational potential in the conservation law. The energy conservation can be reworked as an energy conservation along a magnetic flux tube. Under the assumption of a steady state solar wind, and further assuming that the kinetic energy related to the solar rotation speed is small and that the only dominant energy contribution at $1 \mathrm{AU}$ is the kinetic energy, we find after some algebra the flux of the Alfvén waves:

$$
\begin{aligned}
\left|u_{\mathrm{A}, r}\right| E_{\mathrm{w}}= & \frac{\left(\rho u r^{2}\right)_{1 \mathrm{AU}}}{r^{2}} f_{\mathrm{s}}\left(\frac{u_{1 \mathrm{AU}}^{2}}{2}+\frac{G M_{\odot}}{r}-\frac{\gamma}{\gamma-1} \frac{p_{\mathrm{p}}+p_{\mathrm{e}}}{\rho}\right) \\
& +\kappa_{\mathrm{e}} T_{\mathrm{e}}^{5 / 2} \frac{\partial T_{\mathrm{e}}}{\partial r},
\end{aligned}
$$

where all quantities are taken at the inner boundary $r=$ $1.035 R_{\odot}$ except where $1 \mathrm{AU}$ is indicated by the subscript. In the derivation, we have used the conservation of mass along the flux tube: $\left.\rho u r^{2}\right|_{1 \mathrm{AU}}=\rho u r^{2} /\left.f_{\mathrm{s}}\right|_{1.035 R_{\odot}}$. The mass flux at $1 \mathrm{AU}$ is fixed by setting the proton flux density $n_{\mathrm{p}} u_{\mathrm{r}}$ to $2.7 \times 10^{8} \mathrm{~cm}^{-2} \mathrm{~s}^{-1}$ in the fast wind, consistent with the Ulysses observations of McComas et al. (2000). In Withbroe (1989), the radiative energy balance model was used to explain why the proton flux density at $1 \mathrm{AU}$ is near constant in time with values of approximately $2.7 \pm 0.4 \times 10^{8} \mathrm{~cm}^{-2} \mathrm{~s}^{-1}$ in the fast wind and approximately $3.9 \pm 1.5 \times 10^{8} \mathrm{~cm}^{-2} \mathrm{~s}^{-1}$ in the slow wind. However, since the inner boundary of our solar wind model is higher up in the corona at $r=1.035 R_{\odot}$, this radiative energy balance boundary condition is not applicable in our case. We will instead vary the proton flux density with the ad hoc formula,

$$
n_{\mathrm{p}} u_{\mathrm{r}}=2.7 \times 10^{8} \mathrm{~cm}^{-2} \mathrm{~s}^{-1} \sqrt{\frac{800\left(\mathrm{~km} \mathrm{~s}^{-1}\right)}{u_{1 \mathrm{AU}}\left(\mathrm{km} \mathrm{s}^{-1}\right)}},
$$

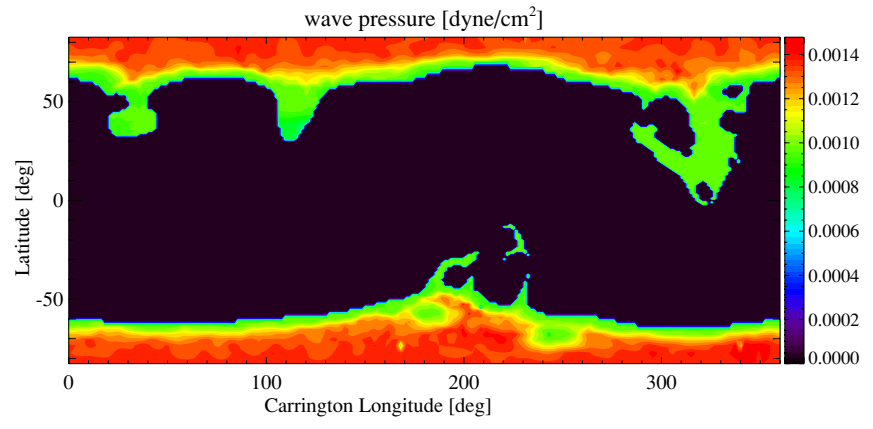

Figure 3. Carrington map for the Alfvén wave pressure at $r=1.035 R_{\odot}$. (A color version of this figure is available in the online journal.)

that more or less fits the above-mentioned fast and slow wind proton flux. Here the latitudinal dependence is given by the WSA velocity at 1 AU.

In general, the electron thermal heat flux in Equation (17) needs to be approximated numerically using the gradient of the electron temperature solution. This means that the Alfvén wave energy density at the inner boundary will depend on the electron temperature solution. It is to be noted that in our solar wind model the Alfvén waves heat only the protons and the electron-proton coupling term in turn heats the electrons, which then modifies the Alfvén wave energy density. However, since the inner boundary is at $r=1.035 R_{\odot}$ where the electron temperature is in the $1 \mathrm{MK}$ range and away from the transition region, the contribution of the heat flux in the coronal hole is small compared to the other terms in Equation (17) and can be neglected in determining the Alfvén wave energy density.

For positive $B_{r}$, only Alfvén waves propagating in the direction of $\mathbf{B}$ emanate from the Sun in our model. For negative $B_{r}$, only Alfvén waves propagating in the direction opposite to $\mathbf{B}$ are launched. In both cases, the waves propagate away from the Sun. In the outlined procedure for obtaining the Alfvén wave energy density at the inner boundary, the waves in our model are effectively only launched from the open field-line region as determined by the PFSS model. Under the action of these waves, the open field of the PFSS model is likely to remain open in the wind model. The same is likely to be true for the closed field-line region. This means that the solar wind model very likely keeps the same open/closed topology of field as the PFSS model.

In Figure 3, the Carrington map of the Alfvén wave pressure is shown for CR2077 at the inner boundary $r=1.035 R_{\odot}$. Here we have used the LDEM density and electron temperature of the previous section in the calculation of the wave pressure. In the closed field-line region, the wave pressure is set to zero at the inner boundary.

\subsection{Model Setup}

The Space Weather Modeling Framework (SWMF) is a flexible and high-performance computational tool for space weather simulations and other space science applications (Tóth et al. 2005). The SWMF combines extensive numerical space science into a single coupled model. The previous SC and $\mathrm{IH}$ models in the SWMF use a spatially varying adiabatic index to account for the acceleration and heating of the plasma (Cohen et al. 2007; Roussev et al. 2003). The version of the SC and IH models, as described in Cohen et al. (2007), uses the WSA model and a Bernoulli integral along the PFSS field lines to determine 


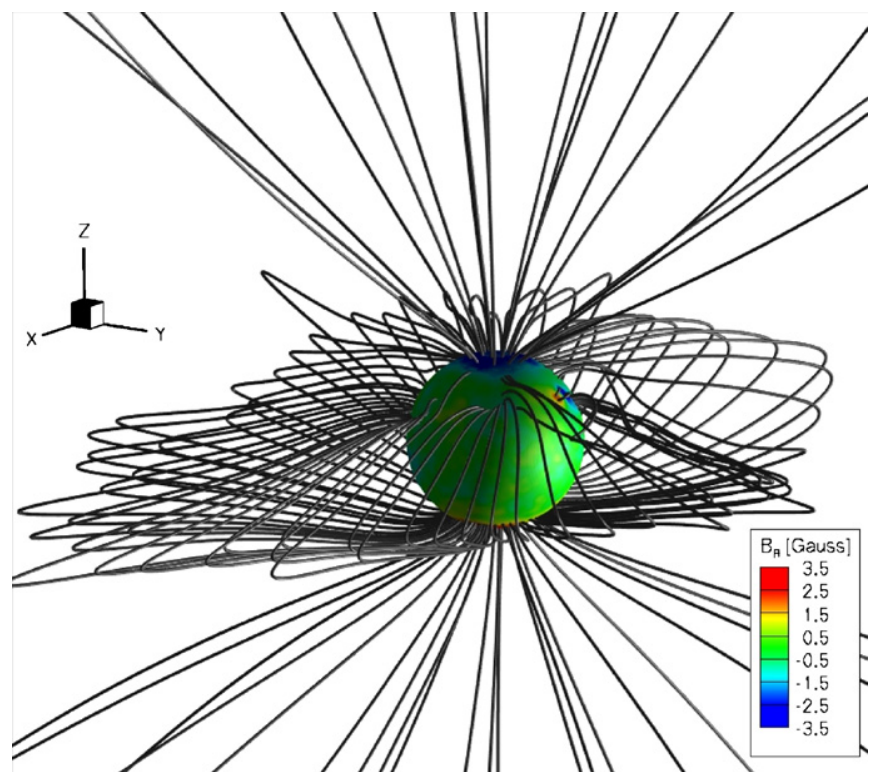

Figure 4. Three-dimensional magnetic field topology showing a few selected field lines of the helmet streamer and coronal hole. The signed radial magnetic field strength at $r=1.05 R_{\odot}$ is shown in color contour.

(A color version of this figure is available in the online journal.)

$\gamma$ in the SC and a uniform $\gamma=1.5$ in the IH. That model was designed to reproduce the observations at $1 \mathrm{AU}$, however, it does have the disadvantage that the reduced $\gamma$ distorts the propagation of CMEs and CME-driven shocks. The newly developed SC and IH models in this paper use $\gamma=5 / 3$ and can be selected within this framework as an alternative to the previous models. Both the old and new SC and IH models are implemented in the MHD BATS-R-US code (Powell et al. 1999) within the SWMF.

For the new SC model, we use a computational domain that covers the ranges $-24 R_{\odot} \leqslant(x, y, z) \leqslant 24 R_{\odot}$, with the Sun at the origin and the rotation axis aligned with the $z$-direction. The domain is decomposed into $4 \times 4 \times 4$ Cartesian grid blocks. The initial grid has five refinement levels. The smallest cell size is approximately $1 / 43 R_{\odot}$ near the Sun and increases toward the outer boundary to a cell size of $0.75 R_{\odot}$. We initialize with Parker's hydrodynamic isothermal solar wind solution (Parker 1958) in combination with the PFSS solution for the magnetic field and relax the system of equations in Section 2.1 in the heliographic rotating frame, which includes the Coriolis and centrifugal forces, subject to the boundary conditions in Section 2.2. To speed up the convergence, local time stepping is used. During the convergence to the steady state solar wind, we twice perform an adaptive mesh refinement of the heliospheric current sheet (HCS). The final number of used cells is typically of the order of 2.5 million.

The IH can be solved in either the rotating or in the inertial frame. In this paper, we use the rotating frame. The domain covers the ranges $-250 R_{\odot} \leqslant(x, y, z) \leqslant 250 R_{\odot}$, with the inner boundary located at $r=16 R_{\odot}$. The inner boundary conditions of the IH model are obtained from the SC model. We refine the grid near the inner boundary of $\mathrm{IH}$ and near the current sheet using $4 \times 4 \times 4$ Cartesian blocks. The smallest cell size is $0.49 R_{\odot}$ and toward the outer boundary the cell size increases to about $3.9 R_{\odot}$. The total number of cells used in the IH amounts to approximately 11.2 million cells.

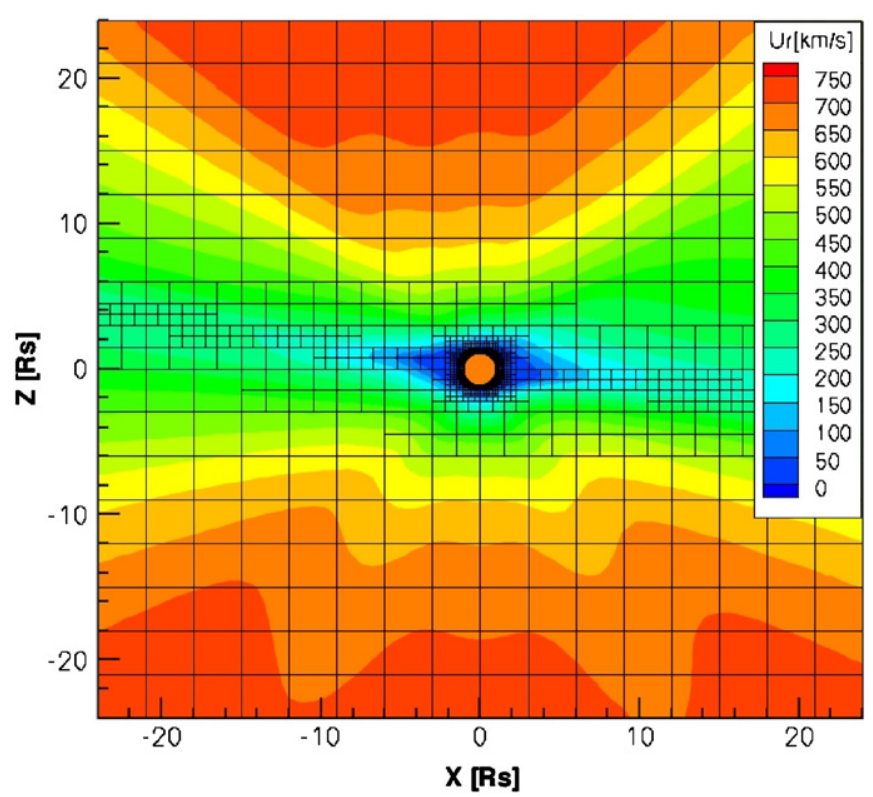

Figure 5. Radial solar wind velocity in a meridional slice. The square boxes represent the grid blocks, showing adaptive mesh refinement near the sun and the current sheet.

(A color version of this figure is available in the online journal.)

\section{RESULTS}

\subsection{Solar Corona}

For this paper, we selected the period CR2077 (2008 November 20 through December 17), which is close to the absolute minimum of sunspots number for solar cycle 23, which occurred in 2008 December according to the National Oceanic and Atmospheric Administration Space Weather Prediction Center. ${ }^{3}$ The initial condition for CR2077 is based on the one-dimensional isothermal solution of Parker combined with a PFSS solution for the magnetic field. We impose on the system the boundary conditions given by the synoptic GONG magnetogram for the radial magnetic field component, density, and temperature obtained from the DEMT analysis, and the Alfvén wave energy density as given by the empirical WSA model in combination with the Bernoulli function along the PFSS field. The parameter $C$ in the Kolmogorov dissipation, Equation (9), is set to $3.4 \times 10^{5} \mathrm{~km} \sqrt{\mathrm{G}}$ in units where $B$ is expressed in Gauss. This value of $C$ does give the best result for CR2077: the density at $1 \mathrm{AU}$ fits the $A C E$ data best, see below. A $3 \mathrm{D}$ view of the magnetic field structure of the steady state solar wind model is shown in Figure 4. The distribution of $B_{\mathrm{r}}$ at $r=1.05 R_{\odot}$ as well as selected magnetic field lines indicating the location of the streamer belt where the plasma is trapped is displayed. Additionally, a few selected open field lines of the coronal holes are shown.

The radial velocity in the $y=0$ plane is shown in Figure 5. Our solar wind model reproduces the bimodal wind solution of the solar minimum with a fast wind above $700 \mathrm{~km} \mathrm{~s}^{-1}$ at high latitude and slow wind below $400 \mathrm{~km} \mathrm{~s}^{-1}$ at low latitude. The streamer belt of zero velocity (except for the syphon flows along the closed field lines) is visible near the Sun. The square boxes show the adaptive mesh blocks indicating the refinement near the Sun and at the HCS. The latter is where the radial magnetic

\footnotetext{
3 http://www.swpc.noaa.gov/SolarCycle
} 

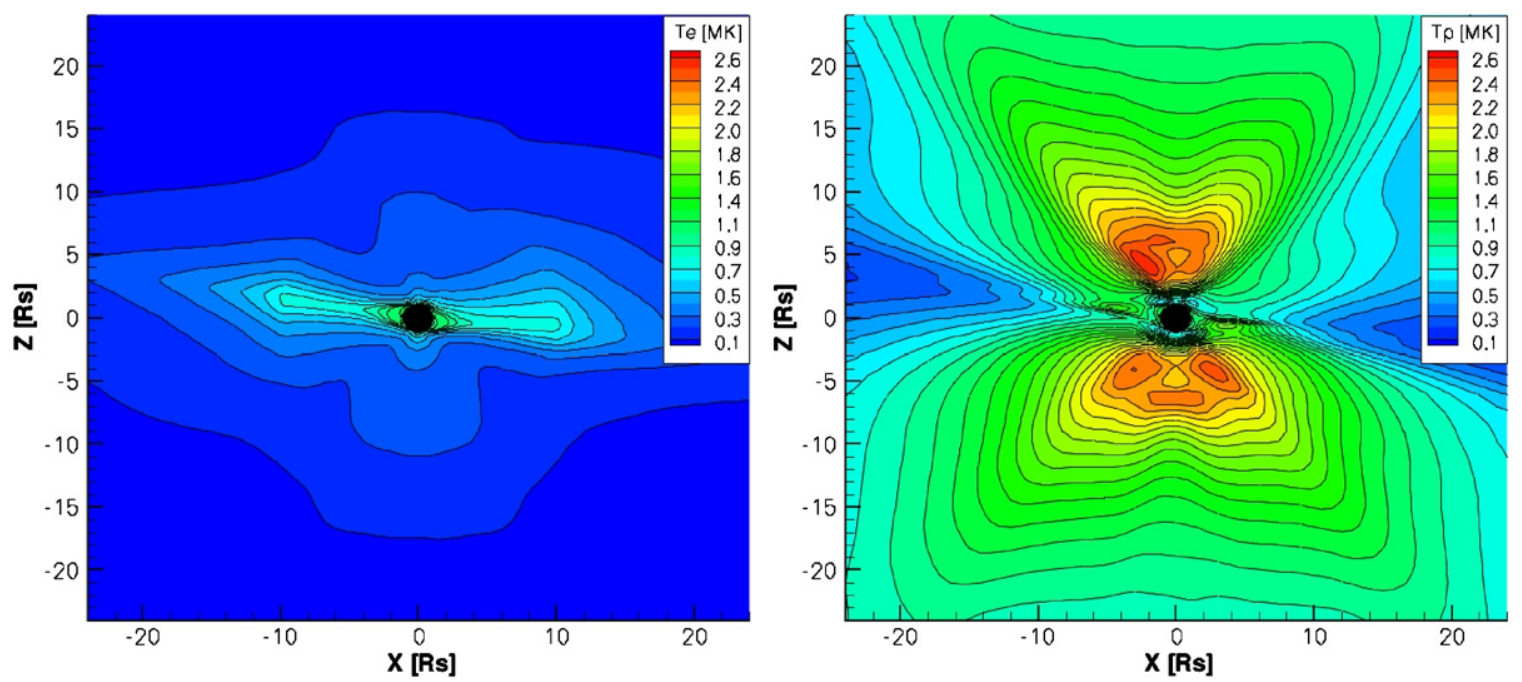

Figure 6. Meridional slice of the SC showing the electron temperature (left panel) and proton temperature (right panel).

(A color version of this figure is available in the online journal.)

field reverses polarity in the slow wind region. This is also the latitude where the plasma is the most dense (not shown).

Figure 6 depicts the electron temperature (left panel) and proton temperature (right panel) in the $y=0$ plane. Very close to the Sun the electrons are heated by the Coulomb collisions with the protons. These collisions decrease with the density so that further away from the Sun the electron and proton temperatures are no longer equalized. In the streamer region and above, the electron temperature maintains a temperature of the order of $1 \mathrm{MK}$ because of the electron heat conduction. However, in the coronal hole the electron temperature decreases due to the cooling by the adiabatic expansion of the plasma. The proton temperature also displays a bimodal structure. The protons are however hotter in the fast wind than the slow wind due to the heating by the dissipation of the Alfvén waves. In our model, the proton temperature reaches a peak of $2.6 \mathrm{MK}$ at about 5 solar radii. From the observations with the Ultraviolet Coronagraph Spectrometer on board the Solar and Heliospheric Observatory, Kohl et al. (2006) give for the solar minimum polar coronal hole an upper and lower estimate for the proton temperature of about 3.5 MK and somewhat below $2 \mathrm{MK}$, respectively. The upper limit was derived from $\mathrm{H}$ I Ly $\alpha$ line width and contains, besides small-scale random motions, also the large-scale nonthermal motions. The lower estimate was obtained from subtracting the nonthermal wave motions to obtain the thermal temperature. Our simulated maximum proton temperature of $2.6 \mathrm{MK}$ is between these two limits.

There are no counterpropagating Alfvén waves in our solar wind model. This statement is equivalent to saying that the wave perturbations in velocity and magnetic field are assumed to be in equipartition everywhere in the corona: $\delta u=\delta B / \sqrt{\mu_{0} \rho}$. This means that the wave perturbation velocity is related to wave energy density by $\delta u=\sqrt{E_{\mathrm{w}} / \rho}$. In Figure 7, the Alfvén wave velocity perturbation is shown along the northern polar coronal hole. At $r=1.035 R_{\odot}$, the velocity perturbation is $44 \mathrm{~km} \mathrm{~s}^{-1}$. The maximum velocity perturbation $274 \mathrm{~km} \mathrm{~s}^{-1}$ is reached at $r=5 R_{\odot}$. This profile looks qualitatively similar to Figure 15 of Cranmer \& van Ballegooijen (2005).

\subsection{Inner Heliosphere}

The 3D IH solution is depicted in Figure 8. A few selected field lines are drawn to show the Parker spiral. In the northern

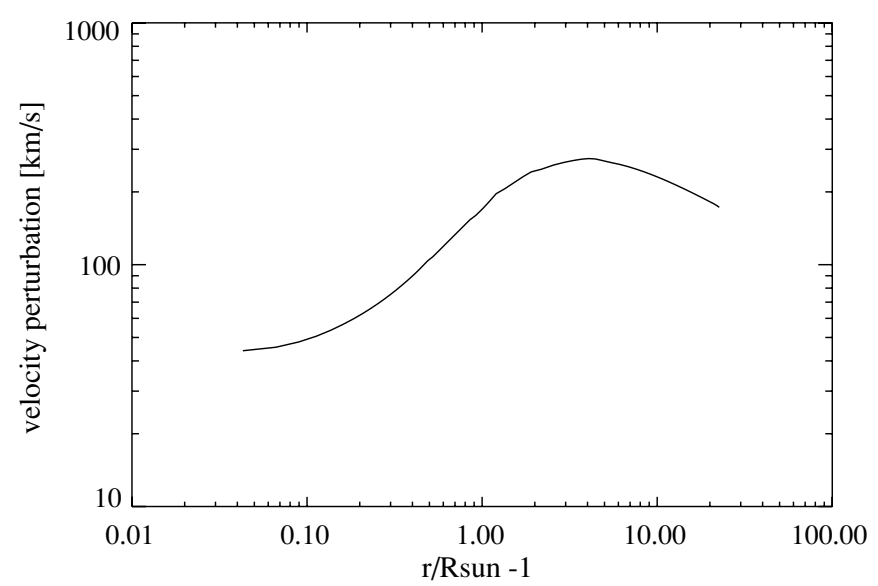

Figure 7. Velocity perturbation amplitude of the Alfvén waves along the north pole.

hemisphere the field lines are spiraling northward, and in the southern hemisphere they spiral southward. The field lines are colored to show the radial velocity. The sign of the radial magnetic field component is shown in black ( - sign) and white (+ sign) in the equatorial plane. This sign reversal is indicative of the warping of the current sheet. For the radial magnetic field component map of CR2077 in Figure 1, negative polarity of the magnetic field is found mostly northward of the equator and positive polarity is mostly southward, so that for negative $B_{\mathrm{r}}$ in the equator the HCS is below the equatorial plane and for positive $B_{\mathrm{r}}$ it is above.

Figure 9 shows the radial velocity (left panel) and number density (right panel) in the equatorial plane. Three fast and slow streams are visible in alternating succession. The plasma is compressed in the regions where the fast stream catches up with the slow stream. At the rear of the fast streams, the plasma is rarified. The resulting effect gives the appearance of three dense spiral arms in the right panel of Figure 9. The interaction regions between the fast and slow streams are known as corotating interaction regions (CIRs). The existence of CIRs is known for several decades and there is an extensive literature on this topic (see, e.g., Jian et al. 2006; Mason et al. 2009, and references therein). Below, we will use these CIRs for the validation study of the new solar wind model. 


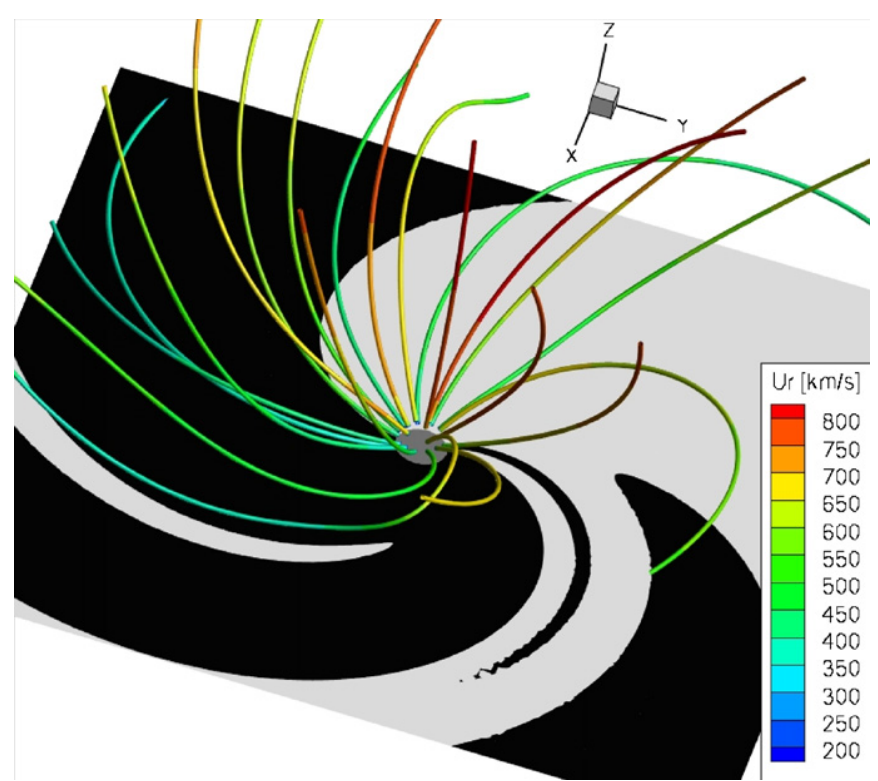

Figure 8. Selected magnetic field lines that show the Parker spiral in 3D. The field lines are colored by the radial velocity. The equatorial plane shows the sign of $B_{\mathrm{r}}$ in black ( - sign) and white (+ sign).

(A color version of this figure is available in the online journal.)

Figure 10 shows the comparison of the simulated synoptic wind with the $A C E$ data at $1 \mathrm{AU}$. We compare the radial velocity (top left panel), proton number density (top right), proton temperature (bottom left), and magnetic field strength (bottom right). The blue line is the hourly averaged $A C E$ data obtained from the Coordinated Data Analysis Web. ${ }^{4}$ The black line is the simulated data in the same time interval of the CR2077. In the bottom left panel, we also show the simulated electron temperature. In the simulated density and radial velocity, there are three CIRs visible. The density peaks are ahead of the velocity peaks as expected, since the CIR plasma compression region ahead of the fast stream is caused by the fast stream catching up with the slow one (see also Figure 9). The locations

4 http://cdaweb.gsfc.nasa.gov

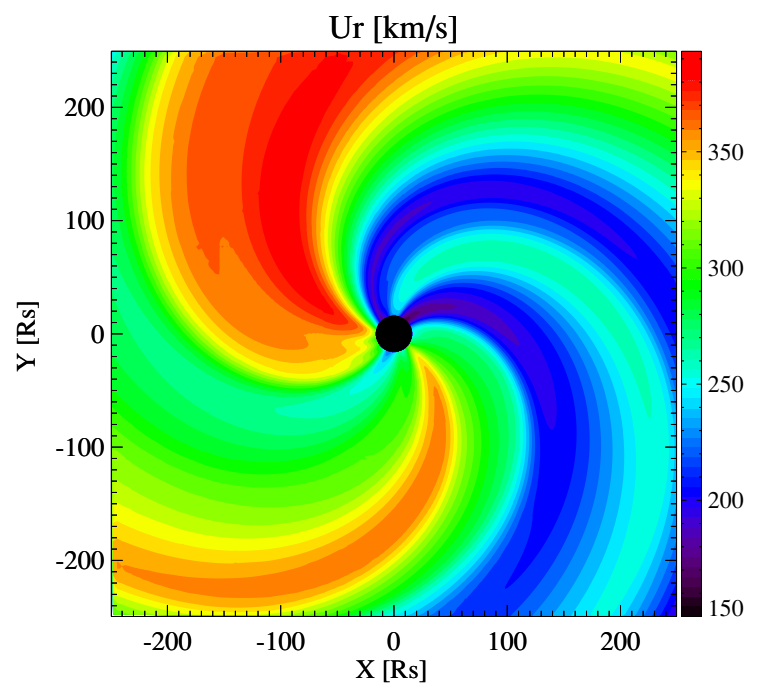

of the peaks are all collocated with those observed by $A C E$. While the amplitude of the density peaks match the $A C E$ data rather well, the radial velocity peaks are relatively smaller. A similar characteristic is evident for both the proton temperature and the magnetic field strength. It is to be noted that we no longer apply an artificial scaling factor of the order of 2.5-4 to the magnetogram to increase the field strength at $1 \mathrm{AU}$ as was done in our previous solar wind model (Cohen et al. 2007). Setting the scaling factor to 1 results in a field strength at $1 \mathrm{AU}$ that is already close to the right order of magnitude. The option of rescaling is still possible and can be used to better match the $A C E$ observations. We also note that the real strength of the new model compared to Cohen et al. (2007) is that the density is in better agreement at $1 \mathrm{AU}$.

The rather low magnitude in velocity and proton temperature is very likely caused by the quite extended slow wind region. This can be seen in Figure 11 where the bimodal nature of the radial velocity and proton temperature is shown in the $y=0$ plane. If the latitudinal extent of the slow wind region were somewhat smaller, then the simulated $A C E$ satellite would be for longer periods of time in the fast wind and larger velocities and proton temperatures would be expected during these times. The reason for the discrepancy with the real in situ data could be twofold: (1) the WSA model provides a velocity distribution at $1 \mathrm{AU}$ and we applied a conservation of energy along the flux tube of the PFSS to the inner boundary $r=1.035 R_{\odot}$ and obtained an approximation for the Alfvén wave energy at this inner boundary. Subsequently, we used this boundary data to calculate the velocity at 1 AU using the 3D Alfvén wave driven solar wind model. However, the simulated 3D solar wind has in general a non-potential magnetic field topology that is different from the PFSS field structure and therefore it is not apparent if the $3 \mathrm{D}$ wind model would obtain the same velocity distribution at $1 \mathrm{AU}$ as the WSA model. (2) Further improvement can be expected with a more advanced treatment of the Alfvén waves by including the counterpropagating Alfvén waves due partial reflection by the inhomogeneity in the density and magnetic field of the solar wind, see, e.g., Chandran et al. (2009b) and Cranmer (2010). This will probably improve the heating and acceleration of the solar wind plasma. Despite these shortcomings, we think

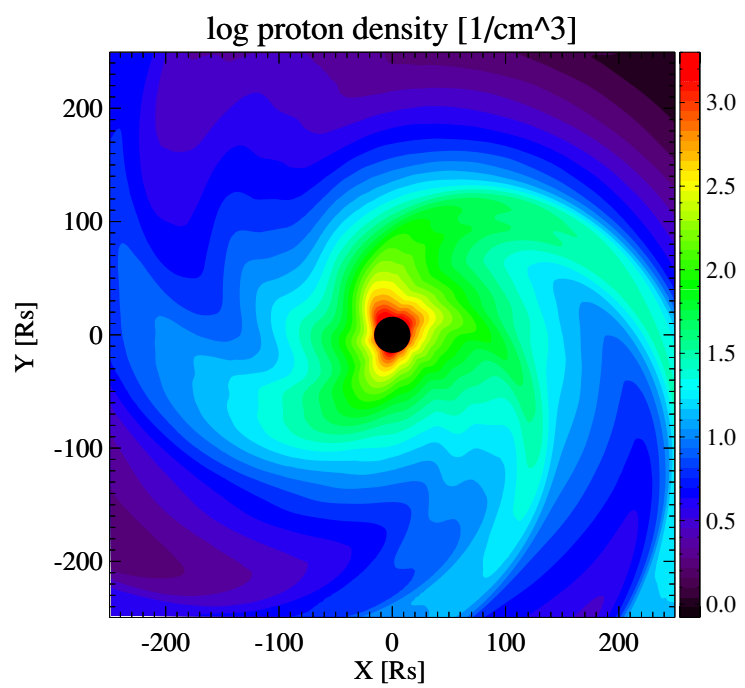

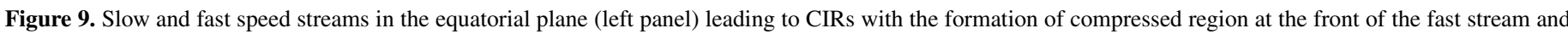
rarefaction at the rear as shown by the density plot (right panel).

(A color version of this figure is available in the online journal.) 

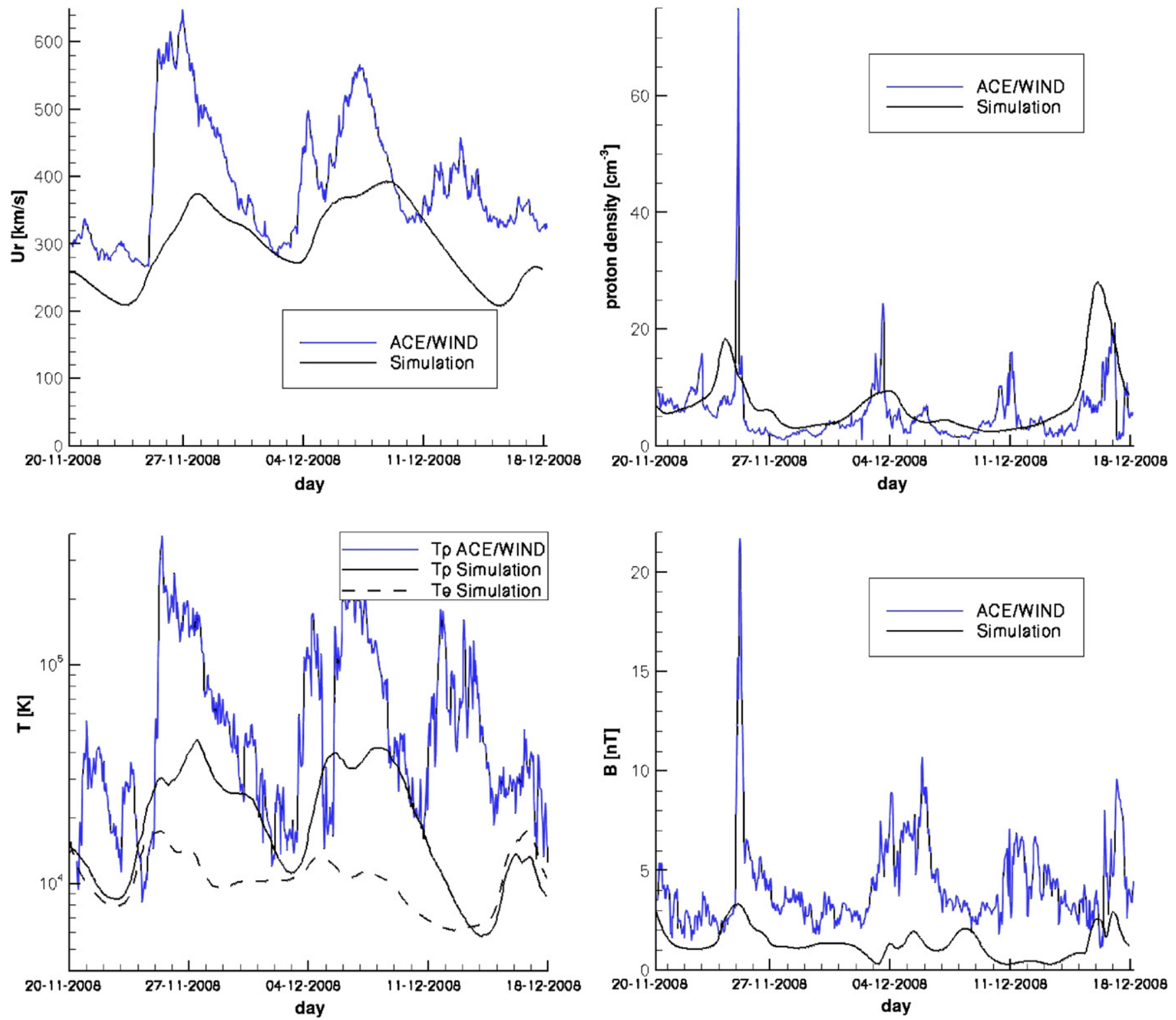

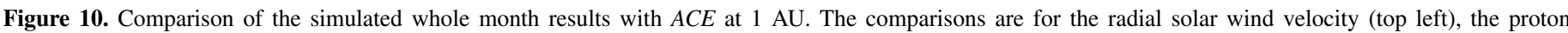

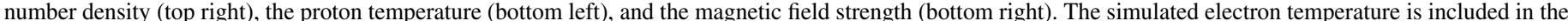
temperature plot.

(A color version of this figure is available in the online journal.)

that our new solar wind model is a step toward physics-based 3D solar wind modeling.

\section{SUMMARY AND CONCLUSIONS}

A new 3D solar wind model is developed in the SWMF that includes WKB Alfvén waves for the acceleration and heating of the plasma. For the wave dissipation, we use the empirical formula of Hollweg (1986) for the Kolmogorov dissipation. We further take into account the separate electron and proton temperatures. This is important because of the infrequent collisions between the electrons and protons away from the Sun. While it is currently still a debate how turbulence partitions energy between the electrons and protons (see, for instance, Stawarz et al. 2009; Breech et al. 2009), we decided to restrict our solar wind model to the heating of the protons by the dissipation of Alfvén waves. We further restrict the anisotropic heat conduction to the electrons only. The system of equations is recast in a (near) conservation form and shock capturing schemes are used to solve for the time evolution of the plasma. This will allow us to model CME-driven shocks with this new solar wind model in future work.

For inner boundary conditions of the solar wind, we use data from observations. Synoptic GONG magnetograms are used for the radial magnetic field, while the electron density and temperature are taken from EUVI/STEREO DEMT. The Alfvén wave pressure near the Sun is obtained from the empirical WSA model for the velocity at $1 \mathrm{AU}$ in combination with the Bernoulli function along the field lines of the PFSS solution.

Despite the fact that our new solar wind model is more physics-based than our previous semi-empirical model with a spatially reduced adiabatic index (Cohen et al. 2007), it still contains several adjustable parameters. One of them is related to the correlation length of the Alfvén waves for the wave dissipation. Changing the source surface radius of the PFSS field will change the size of the streamers and coronal hole boundary. 

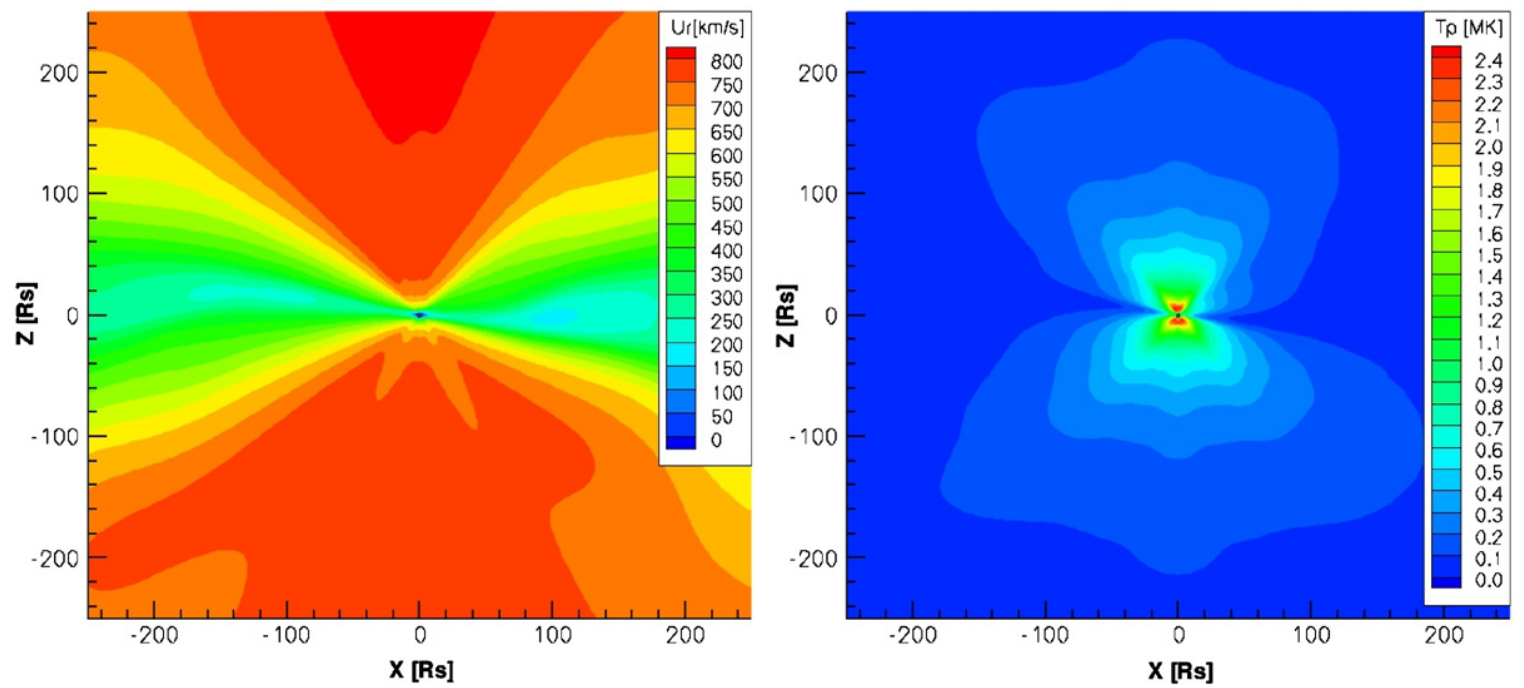

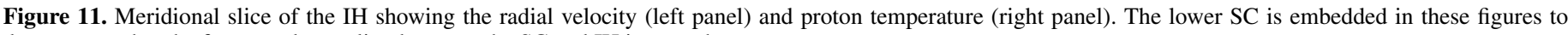
demonstrate that the framework coupling between the SC and $\mathrm{IH}$ is smooth.

(A color version of this figure is available in the online journal.)

This change will also impact the WSA model. The latter also contains free parameters relating how the expansion factor and distance to the coronal hole boundary impact the velocity at 1 AU. Some parameters we did not consider from the onset, for instance the fraction of the Alfvén wave dissipation that channels to the electrons and the efficiency of the Kolmogorov turbulence (this parameter is absorbed in the correlation length of the Alfvén waves).

We performed a validation study of the new 3D solar wind model and the results for CR2077 are encouraging. Three CIRs were found and their time of occurrence at the simulated $A C E$ satellite matches the real $A C E$ observations reasonably well. There is still room for improvement. The velocity, proton temperature, and magnetic field profiles of the simulated $A C E$ data are rather low compared to the real in situ data, especially at times that the $A C E$ satellite is in the fast wind. If the simulated latitudinal extent of the slow wind region were smaller, the simulated $A C E$ trajectory would be for longer periods of time in the fast wind and this would result in higher velocities and temperatures in better agreement with the in situ data. This suggests that further improvements are needed for the heating and acceleration by, for instance, including the counterpropagating Alfvén waves (see, for example, Chandran et al. 2009b; Cranmer 2010). In addition, the low magnetic field strength at $1 \mathrm{AU}$ could be due to the weak polar field of the photospheric magnetograms. The challenges and limitations related to creating global solar magnetic maps are still an open issue (see, e.g., the session on this topic at the Solar Heliospheric and Interplanetary Environment (SHINE) 2006-2010 workshops). Changes in the magnetic maps will definitely have an impact on the velocities at $1 \mathrm{AU}$ obtained from the WSA model. This will in turn modify the Alfvén wave energy density we assign at the inner boundary and therefore impact the heating and acceleration of the solar wind plasma.

The previous solar wind model (Cohen et al. 2007) in the SWMF was a semi-empirical model based on a spatially reduced adiabatic index for the heating and acceleration. While this model was able to reproduce some of the solar wind properties at $1 \mathrm{AU}, \mathrm{CME}$-driven shocks are not well described by it due to its reduced adiabatic index. Our new solar wind model in the SWMF uses $\gamma=5 / 3$ everywhere, is data-driven and physics based. We therefore expect great improvement for the modeling of CMEs and CME-driven shocks.

This work was supported by the IMPACT instrument of NASA's STEREO mission under contract SA4765-26309, by the National Science Foundation under grant ATM 0642309 and by NASA under grant NNX07AC16G. R. A. F. was supported by NASA Heliophysics Guest Investigator award NNX08AJ09G to the University of Michigan. W. B. M. IV was supported by NASA grant LWS NNX09AJ78G. The simulations were performed on the NASA Advanced Supercomputing system Pleiades.

This work utilizes data obtained by the Global Oscillation Network Group (GONG) Program, managed by the National Solar Observatory, which is operated by AURA, Inc. under a cooperative agreement with the National Science Foundation. The data were acquired by instruments operated by the Big Bear Solar Observatory, High Altitude Observatory, Learmonth Solar Observatory, Udaipur Solar Observatory, Instituto de Astrofśica de Canarias, and Cerro Tololo Interamerican Observatory.

\section{REFERENCES}

Altschuler, M. D., \& Newkirk, G. 1969, Sol. Phys., 9, 131

Arge, C. N., Odstrcil, D., Pizzo, V. J., \& Mayer, L. R. 2003, in AIP Conf. Proc. 679, Solar Wind Ten, ed. M. Velli, R. Bruno, \& F. Malara (Melville, NY: AIP), 190

Arge, C. N., \& Pizzo, V. J. 2000, J. Geophys. Res., 105, 10465

Belcher, J. W. 1971, ApJ, 168, 509

Braginskii, S. I. 1965, in Reviews of Plasma Physics, Vol. 1, ed. M. A. Leontovich (New York: Consultants Bureau), 205

Bravo, S., \& Stewart, G. A. 1997, ApJ, 489, 992

Breech, B., Matthaeus, W. H., Cranmer, S. R., Kasper, J. C., \& Oughton, S. 2009, J. Geophys. Res., 114, A09103

Cargill, P. J., \& Priest, E. R. 1980, Sol. Phys., 65, 251

Chandran, B. D. G., \& Hollweg, J. V. 2009a, ApJ, 707, 1659

Chandran, B. D. G., Quataert, E., Howes, G. G., Hollweg, J. V., \& Dorland, W. 2009b, ApJ, 701, 652

Cohen, O., et al. 2007, ApJ, 654, L163

Cranmer, S. R. 2010, ApJ, 710, 676

Cranmer, S. R., \& van Ballegooijen, A. A. 2005, ApJS, 156, 265

Dmitruk, P., Matthaeus, W. H., Milano, L. J., Oughton, S., Zank, G. P., \& Mullan, D. J. 2002, ApJ, 575, 571

Downs, C., Roussev, I. I., van der Holst, B., Lugaz, N., Sokolov, I. V., \& Gombosi, T. I. 2010, ApJ, 712, 1219 
Endeve, E., Holzer, T. E., \& Leer, E. 2004, ApJ, 603, 307

Evans, R. M., Opher, M., Jatenco-Pereira, V., \& Gombosi, T. I. 2009, ApJ, 703, 179

Fang, F., Manchester, W. B., Abbett, W. P., \& van der Holst, B. 2010, ApJ, 714, 1649

Feng, X., Zhou, Y., \& Wu, S. T. 2007, ApJ, 665, 1110

Frazin, R. A., Vásquez, A. M., \& Kamalabadi, F. 2009, ApJ, 701, 547

Harra, L. K., Mandrini, C. H., \& Matthews, S. A. 2004, Sol. Phys., 223, 57

Hartle, R. E., \& Sturrock, P. A. 1968, ApJ, 151, 1155

Hollweg, J. V. 1978, Rev. Geophys. Space Phys., 16, 689

Hollweg, J. V. 1986, J. Geophys. Res., 91, 4111

Howard, R. A., et al. 2008, Space Sci. Rev., 136, 67

Hu, Y. Q., Habbal, S. R., Chen, Y., \& Li, X. 2003a, J. Geophys. Res., 108, 1377

Hu, Y. Q., Li, X., \& Habbal, S. R. 2003b, J. Geophys. Res., 108, 1378

Jacques, S. A. 1977, ApJ, 215, 942

Jian, L., Russell, C. T., Luhmann, J. G., \& Skoug, R. M. 2006, Sol. Phys., 239, 337

Kohl, J. L., Noci, G., Cranmer, S. R., \& Raymond, J. C. 2006, A\&AR, 13, 31

Laitinen, T., Fichtner, H., \& Vainio, R. 2003, J. Geophys. Res., 108, 1081

Lionello, R., Linker, J. A., \& Mikić, Z. 2009, ApJ, 690, 902

Lou, Y. Q. 1994, J. Geophys. Res., 99, 8491

Mason, G. M., Desai, M. I., Mall, U., Korth, A., Bucik, R., von Rosenvinge, R. R., \& Simunac, K. D. 2009, Sol. Phys., 256, 393

McComas, D. J., et al. 2000, J. Geophys. Res., 105, 10419

Mikić, Z., Linker, J. A., Schnack, D. D., Lionello, R., \& Tarditi, A. 1999, Phys. Plasmas, 6, 2217
Ofman, L., \& Davila, J. M. 1995, J. Geophys. Res., 100, 23413

Parker, E. N. 1958, ApJ, 128, 664

Powell, K. G., Roe, P. L., Linde, T. J., Gombosi, T. I., \& DeZeeuw, D. L. 1999, J. Comput. Phys., 154, 284

Roussev, I. I., et al. 2003, ApJ, 595, L57

Ruderman, M. S., Nakariakov, V. M., \& Roberts, B. 1998, A\&A, 338, 1118

Schatten, K. H., Wilcox, J. M., \& Ness, N. F. 1969, Sol. Phys., 6, 442

Sokolov, I. V., Roussev, I. I., Skender, M., Gombosi, T. I., \& Usmanov, A. V. 2009, ApJ, 696, 261

Stawarz, J. E., Smith, C. W., Vasquez, B. J., Forman, M. A., \& MacBride, B. T. 2009, ApJ, 697, 1119

Suzuki, T. K. 2006, ApJ, 640, L75

Tanaka, T. 1994, J. Comput. Phys., 111, 381

Tóth, G., van der Holst, B., \& Huang, Z. 2010, ApJ, submitted

Tóth, G., et al. 2005, J. Geophys. Res., 110, A12226

Tu, C.-Y., \& Marsch, E. 1997, Sol. Phys., 171, 363

Usmanov, A. V., \& Goldstein, M. L. 2006, J. Geophys. Res., 111, A07101

Usmanov, A. V., Goldstein, M. L., Besser, B. P., \& Fritzer, J. M. 2000, J. Geophys. Res., 105, 12675

Vainio, R., Laitinen, T., \& Fichtner, H. 2003, A\&A, 407, 713

van der Vorst, H. A. 1992, SIAM J. Sci. Stat. Comput., 13, 631

Vásquez, A. M., Frazin, R. A., \& Manchester, W. B., IV. 2010, ApJ, 715, 1352

Verdini, A., Velli, M., Matthaus, W. H., Oughton, S., \& Dmitruk, P. 2010, ApJ, 708, L116

Withbroe, G. L. 1988, ApJ, 325, 442

Withbroe, G. L. 1989, ApJ, 337, L49 